\title{
The Neuronal Nitric Oxide Synthase Gene Is Critically Involved in Neurobehavioral Effects of Alcohol
}

\author{
Rainer Spanagel,, ${ }^{1,2}$ Sören Siegmund, ${ }^{1,3}$ Michael Cowen, ${ }^{1}$ Karl-Christian Schroff, ${ }^{1}$ Gunter Schumann, ${ }^{1}$ \\ Magdalena Fiserova, ${ }^{4}$ Inge Sillaber, ${ }^{2}$ Stefan Wellek, ${ }^{5}$ Manfred Singer, ${ }^{3}$ and Jörg Putzke ${ }^{6}$ \\ ${ }^{1}$ Department of Psychopharmacology, Central Institute of Mental Health (CIMH), University of Heidelberg, 68159 \\ Mannheim, Germany, 2Drug Abuse Research Group, Max Planck Institute of Psychiatry, 80804 Munich, Germany, \\ ${ }^{3}$ Department of Medicine IV, University Hospital of Heidelberg, 68167 Mannheim, Germany, 4/nstitute of Pharmacology, \\ Charles University, 10000 Prague, Czech Republic, 5Department of Biostatistics, CIMH, 68159 Mannheim, Germany, and \\ ${ }^{6}$ Department of Medical Neurobiology, University of Magdeburg, 39120 Magdeburg, Germany
}

In the present study, we describe a new role of the neuronal nitric oxide synthase (nNOS) gene in the regulation of alcohol drinking behavior. Mice deficient in the nNOS gene (nNOS -/-) and wild-type control mice were submitted to a two-bottle free-choice procedure with either water or increasing concentrations of alcohol (2-16\%) for 6 weeks. nNOS -/- mice did not differ in consumption and preference for low alcohol concentrations from wild-type animals; however, nNOS -/- mice consumed sixfold more alcohol from highly concentrated alcohol solutions than wild-type mice. Taste studies with either sucrose or quinine solutions revealed that alcohol intake in nNOS -/- and wild-type mice is associated, at least in part, with sweet solution intake but not with the taste of bitterness. When compared with wild-type mice, the nNOS - /- mice were found to be less sensitive to the sedative effects of ethanol as measured by shorter recovery time from ethanol-induced sleep and did not develop rapid tolerance to ethanol-induced hypo- thermia, although plasma ethanol concentrations were not significantly different from those of controls. Our findings contrast with previous reports that showed that nonselective NOS inhibitors decrease alcohol consumption. However, because alcohol consumption was suppressed in wild-type as well as nNOS - /- mice by the NOS inhibitor $N^{G}$-nitro-L-arginine methyl ester, we conclude that the effect of nonselective NOS inhibitors on alcohol drinking is not mediated by nNOS. Other NOS isoforms, most likely in the periphery or other splice variants of the NOS gene, might contribute to the effect of nonselective NOS inhibitors on alcohol drinking. In summary, the nNOS gene is critically involved in the regulation of neurobehavioral effects of alcohol.

Key words: neuronal nitric oxide synthase; nNOS; nNOS splice variants; knock-out mice; alcohol drinking; taste differences; loss of righting reflex; rapid tolerance; NOS inhibitors
Nitric oxide (NO) is an intracellular and extracellular messenger, which is produced by nitric oxide synthase (NOS). There are three NOS genes encoding the respective isoforms: endothelial (eNOS), inducible (iNOS), and neuronal NOS (nNOS). nNOS is a calcium/calmodulin-dependent enzyme that was first found in neurons (Bredt et al., 1990). Evidence from previous studies has implicated the nNOS-NO pathway in the modulation of CNSmediated drug effects. Thus, inhibition of NOS has been shown to influence the development of tolerance (Khanna et al., 1993, 1995; Kolesnikov et al., 1993, 1997) and sensitization (Itzhak et al., 1998; Itzhak and Martin, 2000) to several drugs of abuse, including alcohol. Beside these findings, two other lines of evidence have prompted us to study the role of the nNOS gene in the regulation of neurobehavioral effects of alcohol:

First, it has been shown that inhibition of NO formation reduces voluntary alcohol consumption in rats. Thus, the administration of either $N^{\mathrm{G}}$-nitro-L-arginine (L-NNA) or $N^{\mathrm{G}}$-nitro-Larginine methyl ester (L-NAME) (both compounds inhibit all

Received March 22, 2002; revised June 24, 2002; accepted July 1, 2002.

This work was supported by Bundesministerium für Bildung und Forschung Grants FKZ 01GS0117 (R.S.), FKZ EB 01011300/6 (R.S.), and FKZ 2766A/0087H (J.P.).

Correspondence should be addressed to Rainer Spanagel, Department of Psychopharmacology, Central Institute of Mental Health, J5, 68159 Mannheim, Germany. E-mail: spanagel@zi-mannheim.de.

Copyright (C) 2002 Society for Neuroscience $\quad 0270-6474 / 02 / 228676-08 \$ 15.00 / 0$ isoforms of NOS) attenuated alcohol consumption in two lines of alcohol-preferring rats (Rezvani et al., 1995), in Sprague Dawley rats selected for high alcohol intake (Calapai et al., 1996), and in rats that drank alcohol chronically in combination with L-NNA (Lallemand and De Witte, 1997). Together, these experiments show that inhibition of NOS reduces alcohol self-administration; however, at the moment, it is not clear which isoform of NOS is involved in the action of nonselective NOS inhibitors during alcohol consumption.

Second, numerous studies have shown that the glutamatergic system is involved in the mediation of acute and chronic alcohol effects (Tabakoff and Hoffman, 1996; Tsai and Coyle, 1998). The glutamatergic system in turn is strongly linked to the NO pathway (Bredt and Snyder, 1994). Thus, stimulation of glutamatergic NMDA receptors lead to calcium influx, and binding of calcium to calmodulin activates nNOS, which produces NO. Thus, the close link between the glutamatergic-NMDA receptor system and NO production suggests that the nNOS gene is also involved in the modulation of acute and chronic effects of alcohol. This suggestion is further supported by in vitro studies: acute ethanol treatment inhibits NMDA-stimulated NOS activity in cortical neurons (Chandler et al., 1994), whereas chronic ethanol treatment increases NMDA-stimulated NO formation in this preparation (Chandler et al., 1997).

These lines of evidence (the effects of nonselective NOS inhib- 
itors on alcohol drinking and the close association of NMDA receptors and nNOS) have led us to hypothesize that the nNOS gene might be critically involved in the regulation of alcohol drinking behavior. To this end, we studied alcohol drinking behavior in mice deficient in the nNOS $\alpha$ isoform (Huang et al., 1993; Huang and Lo, 1998). We further investigated whether nNOS $-/-$ mice and wild-type mice would differ in terms of sedative-hypnotic effects of an acutely administered high dose of ethanol. This experiment was of interest because it has been proposed that initial sensitivity to ethanol can be negatively correlated with subsequent ethanol intake in humans (Schuckit, 1994; Schuckit and Smith, 1996), as well as in rodents (Thiele et al., 2000). Another phenomenon that often occurs in the course of chronic alcohol intake is tolerance, and it has been shown that NO formation is involved in the development of tolerance to ethanol (Khanna et al., 1993, 1995). Therefore, we also studied the induction of rapid tolerance to ethanol in nNOS knock-out mice.

\section{MATERIALS AND METHODS}

Animals. Ninety-seven homozygote nNOS knock-out mice and 100 wildtype mice were obtained from our breeding colony at the Otto-vonGuericke University of Magdeburg (Magdeburg, Germany). The foundation stock of these animals was initially established at the Massachusetts General Hospital (Boston, MA). The nNOS gene mutation was generated by homologous recombination (Huang et al., 1993). The genetic background is on a combination of the $129 \mathrm{X} 1 / \mathrm{SvJ}$ and $\mathrm{C} 57 \mathrm{BL} / 6 \mathrm{~J}$ strains with a predominance of $\mathrm{C} 57 \mathrm{BL} / 6 \mathrm{~J}$, because mice were backcrossed for three generations into $\mathrm{C} 57 \mathrm{BL} / 6 \mathrm{~J}$ and then intercrossed to obtain knock-out mice and wild-type littermates (Azad et al., 2001). Further backcrossing was not possible because, after the third generation, almost no offspring were obtained any more.

At the beginning of the experiments, the mice were 2-3 months old. All animals were housed individually in standard (type 2) hanging rodent cages with food and water available ad libitum. Artificial light was provided daily from 6:00 A.M. until 6:00 P.M., and room temperature and humidity were kept constant (temperature, $22 \pm 1{ }^{\circ} \mathrm{C}$; humidity, $55 \pm$ $5 \%$ ). The experiments were approved by the Committee on Animal Care and Use of the relevant local governmental body and performed following the German Law on the Protection of Animals.

Drugs. Ethanol-drinking solutions were made up from $96 \%$ ethanol diluted with tap water to the different concentrations. For injections, $96 \%$ ethanol was diluted with $0.9 \%$ saline to a $20 \%(\mathrm{v} / \mathrm{v})$ solution. For oral application, $96 \%$ ethanol was diluted with water to a $12 \%(\mathrm{v} / \mathrm{v})$ solution. Quinine HCl, sucrose, and L-NAME were obtained from Sigma (Deisenhofen, Germany) and were either diluted with tap water or with $0.9 \%$ saline for injections.

Oligonucleotide probes. The following 45-mer synthetic oligonucleotide probes for radioactive in situ hybridization to specific nNOS splice forms were used (for details, see Eliasson et al., 1997; Putzke et al., 2000): (1) nNOS $\alpha$ probe corresponding to residues $99-143$ of exon 2, (2) nNOS $\beta$ probe corresponding to the junction of exons $1 \mathrm{a}$ and 3 , and (3) nNOS $\gamma$ probe corresponding to the junction of exons $1 \mathrm{~b}$ and 3 .

The oligonucleotide probes were $3^{\prime}$-labeled $\left(37^{\circ} \mathrm{C}, 5 \mathrm{~min}\right)$ to specific activities of $2 \times 10^{6} \mathrm{cpm} / \mathrm{pmol}$ using terminal deoxynucleotide transferase (EC 2.7.7.31; Boehringer Mannheim, Mannheim, Germany) and $\left[\alpha-{ }^{35}\right.$ S $]$ dATP $(1000-1500 \mathrm{Ci} / \mathrm{mmol}$; DuPont NEN, Dreieich, Germany) according to the protocol of the manufacturer.

In situ hybridization. Four alcohol-naïve nNOS -/- and wild-type mice were decapitated; brains were removed and immediately frozen on dry ice. Horizontal slices $(14 \mu \mathrm{m})$ were sectioned on precoated slides, fixed with $4 \%$ paraformaldehyde, and stored under alcohol until used. Labeled oligonucleotide probes were then hybridized overnight at $42^{\circ} \mathrm{C}$ in hybridization buffer ( $50 \%$ formamide, $4 \times \mathrm{SSC}$, and $10 \%$ dextran sulfate) to the brain sections. Sections were then washed $\left(1 \times \mathrm{SSC}, 30 \mathrm{~min}, 55^{\circ} \mathrm{C}\right)$, dried, and exposed to x-ray film (Amersham Biosciences, Braunschweig, Germany) for 8 weeks and afterward dipped in photoemulsion. This was followed by an exposure for 2 months, developing with D19 (Eastman Kodak, Rochester, NY), and counterstaining with cresyl violet. Because carbon-14 shows nearly the same activity as sulfur-35, coexposed $\left[{ }^{14} \mathrm{C}\right]$ microscales (Amersham Biosciences) were used to reveal a log-log linear relationship between radioactivity and optical density. Specificity of hybridization was ascertained by the incubation of parallel sections with buffer containing both labeled and a 100 -fold excess of unlabeled probe. These procedures resulted in images indistinguishable from background.

Alcohol self-administration. After 1 week of habituation to the animal room, mice were given continuous access ad libitum to two bottles of tap water for 1 week to study initial side preferences. Then, animals had the free choice between tap water and a $2 \%(\mathrm{v} / \mathrm{v})$ alcohol solution for $3 \mathrm{~d}$. For days $4-6$, the ethanol concentration was increased to $4 \%$, on days 7-16 it was increased to $8 \%$, on days $17-26$ it was increased to $12 \%$, and on days $27-46$ it was increased $16 \%$. Spillage and evaporation were minimized by the use of self-made glass cannulas in combination with a small plastic bottle (Techniplast, Milan, Italy). Under these conditions, ethanol concentration in a given solution stayed constant for at least 1 week, when measured with an alcoholometer (GECO, Gering, Germany). Bottles were weighed daily at 10:00 A.M., and all drinking solutions were renewed every $3 \mathrm{~d}$. For days 1-6, the positions of the two bottles were changed daily, and, for days 7-46, the positions of the two bottles were changed each $3 \mathrm{~d}$ to avoid side preferences.

After the measurement of basal alcohol intake, mice received for an additional 2 weeks water and $16 \%$ ethanol. Wild-type and knock-out animals, respectively, were then divided into two groups ( $n=8$ per group), which were matched according their alcohol intake and preference. Animals were injected with either L-NAME $(25 \mathrm{mg} / \mathrm{kg}$, i.p.) or saline for $2 \mathrm{~d}$. Injections were given once per day at 10:00 A.M.

Taste preference tests. Alcohol-naïve and alcohol-experienced mice were used for these tests. A pilot study showed that alcohol selfadministration for 2 months had no influence on subsequent intake of sweet- or bitter-tasting solutions when compared with age-matched alcohol-naïve mice. Therefore, the taste preference tests were performed in alcohol-naïve mice: sucrose $(0.5,2.5$, and $5 \% \mathrm{w} / \mathrm{v})$ and quinine $(0.01$ and $0.02 \mathrm{~mm}$ ) solution intake was measured in a two-bottle free-choice test (sucrose or quinine against water). A test lasted for $6 \mathrm{~d}$, bottles were weighed every $3 \mathrm{~d}$, and the position of the bottles was then changed.

Measurement of loss of righting response. The procedure for measuring the duration of loss of righting response was similar to that described by Harris et al. (1995). Mice were administered an intraperitoneal injection of ethanol $(20 \% \mathrm{v} / \mathrm{v})$ at a dose of 2.5 and $4.5 \mathrm{gm} / \mathrm{kg}$ body weight. When animals became ataxic, they were placed on their back in a V-shaped paper trough, and the time was recorded until the righting response. Animals were judged to have regained their righting response when they could right themselves three times within $30 \mathrm{sec}$. The observer was blind to the experimental design.

Hypothermia test and rapid tolerance measurements. Because repeated measurement of rectal temperature always induces changes in core temperature (Spanagel et al., 1996), which in turn leads to misinterpretation of data, we used an infrared thermometer (Infratherm, Boston, MA) to measure ventral surface temperature. Pilot studies indicated that changes in core body temperature closely correlate to changes in ventral surface temperature measured by the infrared thermometer.

For the measurement of rapid tolerance to ethanol-induced hypothermia, alcohol-naïve nNOS $-/-$ and wild-type mice were randomly divided into three separate groups of eight to nine animals. On day 1 at 10:00 A.M., the first two groups received saline, and the remaining group received $3.5 \mathrm{gm} / \mathrm{kg}$ ethanol intraperitoneally in their home cage. Eight hours later, the first two groups received saline again, and group 3 received $2 \mathrm{gm} / \mathrm{kg}$ ethanol intraperitoneally. On day 2 at 10:00 A.M., basal body temperature was measured for all animals, and then group 1 received saline, and groups 2 and 3 received $3.5 \mathrm{gm} / \mathrm{kg}$ ethanol intraperitoneally. After $30 \mathrm{~min}$, the temperature was determined again. A pilot study with $\mathrm{C} 57 \mathrm{BL} / 6 \mathrm{~J}$ mice was used to determine the optimal injection schedule and dosing for the development of rapid tolerance (Crabbe et al., 1979). Usually, 30 min after the ethanol injection on day 2, a maximal effect of rapid tolerance occurs.

Blood alcohol determination. Alcohol-naïve wild-type and nNOS - / mice were injected intraperitoneally with $3.5 \mathrm{gm} / \mathrm{kg}$ ethanol. Blood alcohol levels were measured by drawing blood samples $(25-30 \mu \mathrm{l})$ from the tip of the tail at various time points after injection $(30,60,90,120$, and $240 \mathrm{~min}$ ). Blood alcohol levels were also determined in some animals during the alcohol self-administration procedure. Blood alcohol content was determined by the nicotinamide adenine dinucleotide phosphate enzyme spectrophotometric method (Sigma).

Statistical analysis. In all figures, columns represent arithmetic means, and error bars represent SEM. Significance testing was based on ordinary $t$ statistics for paired and unpaired samples throughout. In all compari- 

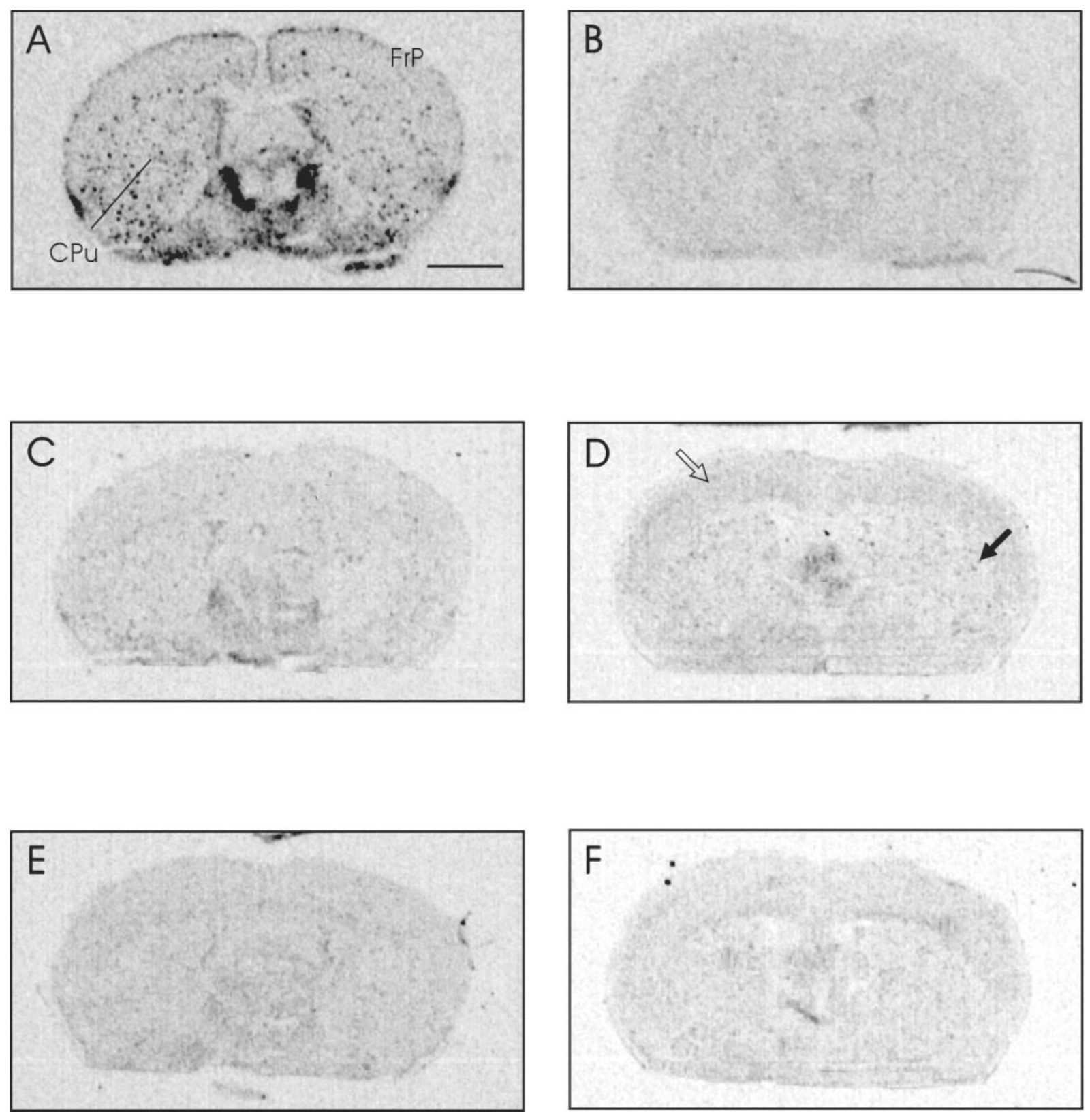

Figure 1. In situ hybridization analysis of $\mathrm{nNOS} \alpha, \beta$, and $\gamma$ mRNA levels in the ventral striatum in wild-type and knock-out animals. Autoradiographs depicting the in situ hybridization of nNOS splice variant probes $\alpha(A, B), \beta(C, D)$, and $\gamma(E, F)$ in horizontal brain sections in wild-type mice $(A$, $C$, $E)$, and $\operatorname{NOS} \alpha-/-$ mice $(B, D, F)$. Scale bar, $10 \mathrm{~mm}$. nNOS $\alpha$ mRNA signals were completely abolished in knock-out mice, whereas nNOS $\beta$ and nNOS $\gamma$ mRNA were still detectable. Moreover, nNOS $\beta$ mRNA seems to be slightly increased in nNOS $\alpha-/-$ animals, especially within striatum ( filled arrow) and cortex (open arrow). $\mathrm{CPu}$, Caudate putamen; $\mathrm{Fr} P$, frontoparietal cortex.

sons relating to parallel groups of animals, we allowed for heteroskedasticity by adjusting the number of degrees of freedom according to the formula of Welch. Occasionally, the test statistics were computed with data obtained from multivariate observations by applying suitable transformations in a preprocessing step. This was the case with the comparison between wild-type and nNOS $-/-$ mice with respect to speed of elimination of ethanol from blood (see Fig. 7). At the intra-individual level, elimination speed was measured by means of ordinary linear regression. Similarly, percentage ethanol intake (see Fig. 4) was calculated with respect to individual rather than averaged baseline.

Control of multiple type I error risk. For the majority of experiments presented in this paper, statistical analysis entailed the assessment of several (up to 36) $p$ values. In all these cases, the multiple type I error risk was kept below $5 \%$ by applying the sequentially rejective procedure of Holm (1979). Accordingly, any significance statement made in Results refers to an experiment rather than comparisonwise type I error risk bounded by $5 \%$. Of course, this implies that a considerable number of $p$ values $\leq 0.05$ have to be declared nonsignificant. In cases in which the multiplicity of pairwise comparisons to be performed refers to contrasts between specific cells of a factorial layout, Holm's procedure replaces more traditional techniques, such as so-called post hoc tests presupposing a significant result of a suitable global test for homogeneity.

\section{RESULTS}

nNOS splice variant gene expression in the brain of wild-type and nNOS -I- mice

nNOS is subject to alternative splicing. Several transcripts have been described; however, the nNOS $\alpha$ variant accounts for most of the catalytic activity in the brain because nNOS $\alpha-/-$ mice, which were used in the present study, display $\sim 95 \%$ reduction in 


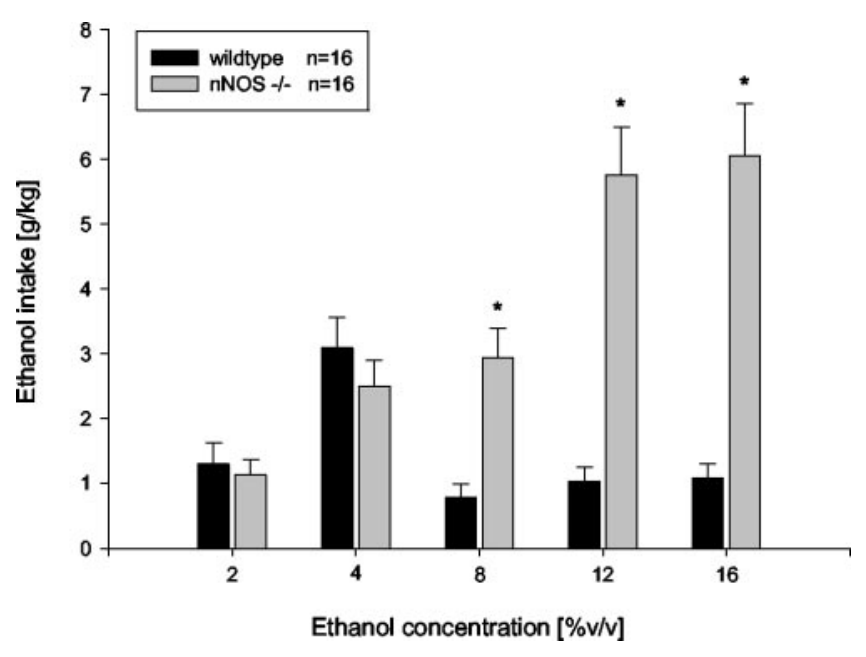

Figure 2. Acquisition of alcohol self-administration in nNOS knock-out and wild-type mice. Mice had the free choice between tap water and a $2 \%$ (v/v) alcohol solution for $3 \mathrm{~d}$. For days 4-6, the ethanol concentration was increased to $4 \%$, for days $7-16$ it was increased to $8 \%$, for days $16-25$ it was increased to $12 \%$, and for days $25-44$ it was increased to $16 \%$. Bars show mean + SEM ethanol intake per day in grams per kilogram body weight. ${ }^{*} p<0.05$ indicates a significant difference between wild-type and nNOS $-/-$ mice in ethanol consumption per day.

NOS catalytic activity (Huang et al., 1993). Two alternatively spliced variants of nNOS, $\beta$ and $\gamma$, still persist in the nNOS $\alpha-/-$. We compared localization of $\mathrm{nNOS} \alpha, \beta$, and $\gamma$ variants by in situ hybridization in wild-type and knock-out mice. nNOS $\alpha$ mRNA is heterogeneously expressed in the forebrain of wild-type mice, following the distribution pattern that was described previously (Eliasson et al., 1997, Putzke et al., 2000), e.g., in the bed nucleus of the stria terminalis, the lateral septum, and hypothalamic regions. Furthermore, a characteristic spot-like distribution pattern was detected in the mesolimbic system, especially within caudate putamen and the anterior cingulate cortex (Fig. 1A). nNOS $\beta$ mRNA is coexpressed especially in the caudate putamen, frontoparietal cortex, and hypothalamic areas (Fig. 1C). Only very low amounts of nNOS $\gamma$ mRNA were detected (Fig. 1E). nNOS $\alpha$ mRNA signals are completely abolished in knock-out mice, whereas nNOS $\beta$ mRNA was still detectable within caudate putamen, as well as the frontoparietal cortex in nNOS $\alpha$ knock-out mice (Fig. $1 B, D$ ). Moreover, compared with wild-type mice, mRNA levels of nNOS $\beta$ seem to be moderately increased in the dorsal and ventral part of the striatum of knock-out mice (Fig. $1 D)$. Like in wild-type animals, only very low abundant nNOS $\gamma$ mRNA could be detected within knock-out animals (Fig. $1 F$ ).

\section{nNOS -/- mice drink more alcohol from highly concentrated alcohol solutions than wild-type mice}

Mice showed no overall initial side preference when they were offered continuous ad libitum access to two bottles of tap water for 1 week. This initial side preference test ensured that subsequent alcohol preference tests were not influenced by an initial side preference. Figure 2 shows the ethanol intake of nNOS -/and wild-type mice in a two-bottle free-choice procedure with increasing concentrations of ethanol. Although no significant difference in ethanol intake between knock-out and wild-type animals was observed for low ethanol concentrations, marked differences occurred at higher concentrations. Ethanol preference data revealed a similar pattern (data not shown). Statistical analyses revealed significant differences between nNOS $-/-$ and
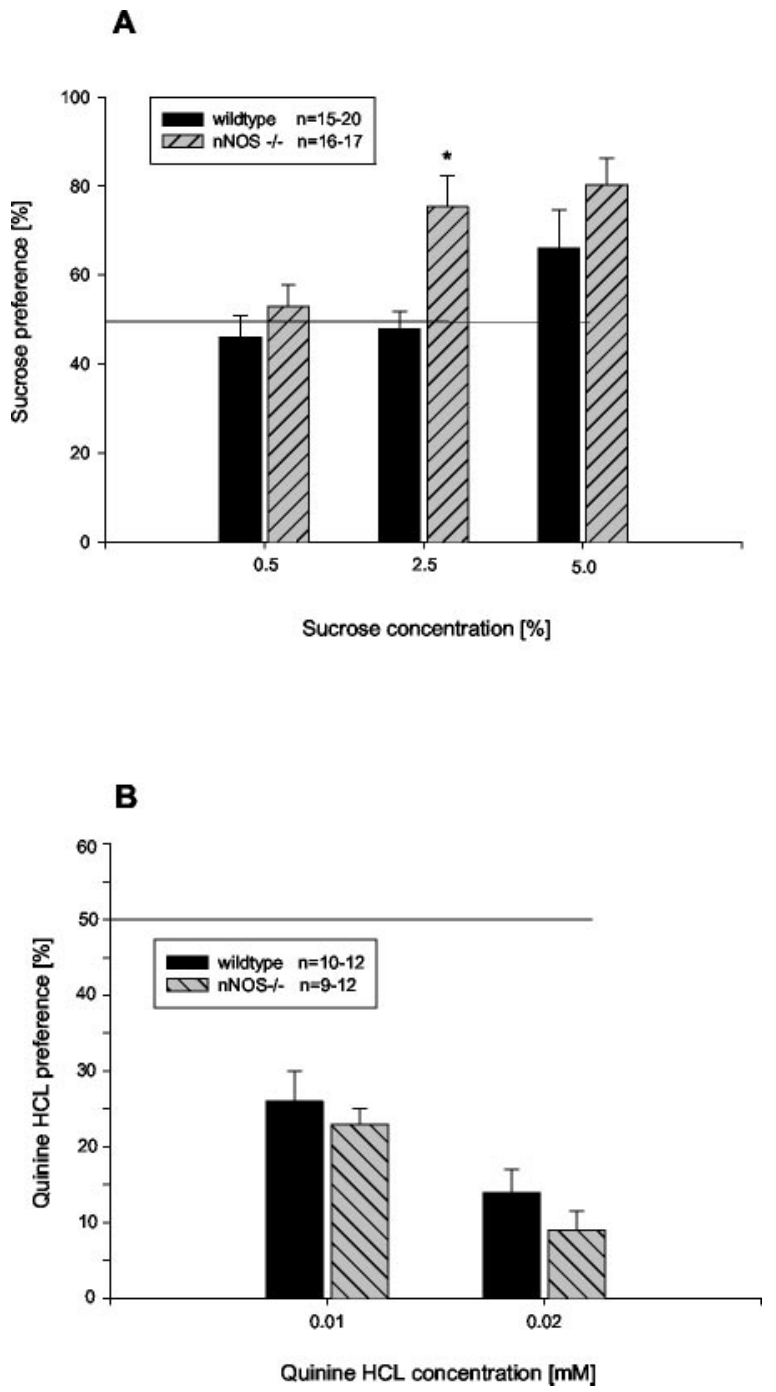

Figure 3. $A$, Sucrose preference in nNOS knock-out and wild-type mice. $B$, Quinine aversion in nNOS knock-out and wild-type mice. Sucrose $(0.5$, 2.5 , and $5 \% \mathrm{w} / \mathrm{v})$ and quinine $(0.01$ and $0.02 \mathrm{~mm})$ solution intake was measured in a two-bottle free-choice test in alcohol-naïve mice. Bars show mean \pm SE sucrose or quinine preference. ${ }^{*} p<0.05$ indicates a significant difference between wild-type and nNOS knock-out mice.

wild-type animals for the voluntary intake of $8 \%(p=0.0003)$, $12 \%(p<0.0001)$, and $16 \%$ ethanol $(p<0.0001)$. At these concentrations, mean consumption was at least six times as large in nNOS $-/-$ as in wild-type mice. The high alcohol intake in nNOS $-/-$ mice resulted in pharmacologically meaningful blood alcohol levels (up to $58 \mathrm{mg} / \mathrm{dl}$ ); however, in wild-type mice, no blood alcohol levels could be determined.

Figure $3 A$ shows a concentration-dependent increase in sucrose preference in wild-type and nNOS $-/-$ animals. Statistical analysis revealed, however, that this increase was more pronounced in the knock-out mice. At an intermediate concentration of $2.5 \%$ sucrose, $\mathrm{nNOS}-1-$ mice had a significantly $\left(t_{(26.7)}=3.05 ; p=\right.$ $0.0051)$ higher preference than wild-type animals. No significant difference between the genotypes could be found in the preference for quinine-containing solutions $\left(0.01 \mathrm{mM}, t_{(20.9)}=0.47, p=\right.$ $\left.0.6404 ; 0.02 \mathrm{~mm}, t_{(17.0)}=0.90, p=0.3812\right)$. Thus, $\mathrm{nNOS}-/-$ and wild-type animals showed a similar concentration-dependent aversion to quinine (Fig. 3B). 

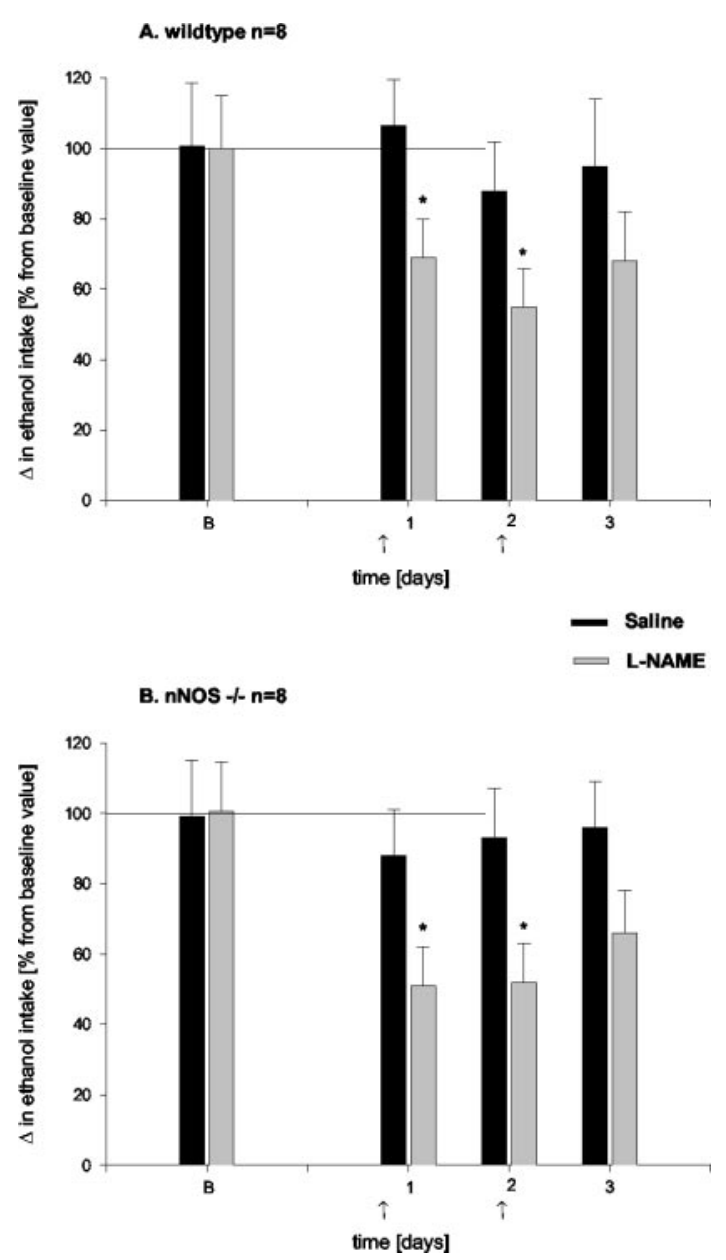

Figure 4. Alcohol intake in wild-type $(A)$ and nNOS knock-out $(B)$ mice after treatment with the NOS inhibitor L-NAME. Because basal alcohol intake differed between both genotypes, all values were normalized and are now given as percentage. L-NAME was injected at days 1 and 2 (indicated by $\uparrow$ ), and bars represent the difference in alcohol intake + SE compared with the basal intake (indicated by $B$ ). ${ }^{*} p<0.05$ indicates a significant difference from basal intake.

In an additional experiment, the effect of the NOS inhibitor L-NAME on alcohol intake in wild-type mice and nNOS -/mice was measured. L-NAME treatment for $2 \mathrm{~d}$ significantly decreased alcohol intake by $>40 \%$ in the knock-out group ( $1 \mathrm{~d}$, $t_{(10.4)}=4.42, p=0.0012 ; 2 \mathrm{~d}, t_{(10.1)}=4.99, p=0.0005 ; 3 \mathrm{~d}$, $\left.t_{(9.25)}=3.49, p=0.065\right)$. Similar effects were seen in wild-type animals; thus, the effect of L-NAME was significant at the multiple $5 \%$ level at day 1 and day 2 of L-NAME treatment $\left(t_{(11.2)}=\right.$ $3.42, p=0.0056 ; 2 \mathrm{~d}, t_{(10.4)}=2.48, p=0.0317 ; 3 \mathrm{~d}, t_{(10.8)}=1.98$, $p=0.0738$ ) (Fig. 4).

\section{nNOS -I- mice are less sensitive to alcohol and do not develop tolerance}

It has been shown in humans as well as animals, including mice, that high levels of alcohol drinking are often associated with resistance to the physiological effects of this drug (Schuckit, 1994; Schuckit and Smith, 1996; Thiele et al., 2000). Because nNOS knock-out mice consumed much more alcohol than wild-type mice, we hypothesized that nNOS $-/-$ mice should be less sensitive to the sedative and hypnotic effects of high ethanol doses. Indeed, at both doses (2.5 and $4.5 \mathrm{gm} / \mathrm{kg})$ tested, nNOS $-/-$ mice regained their righting reflex significantly sooner

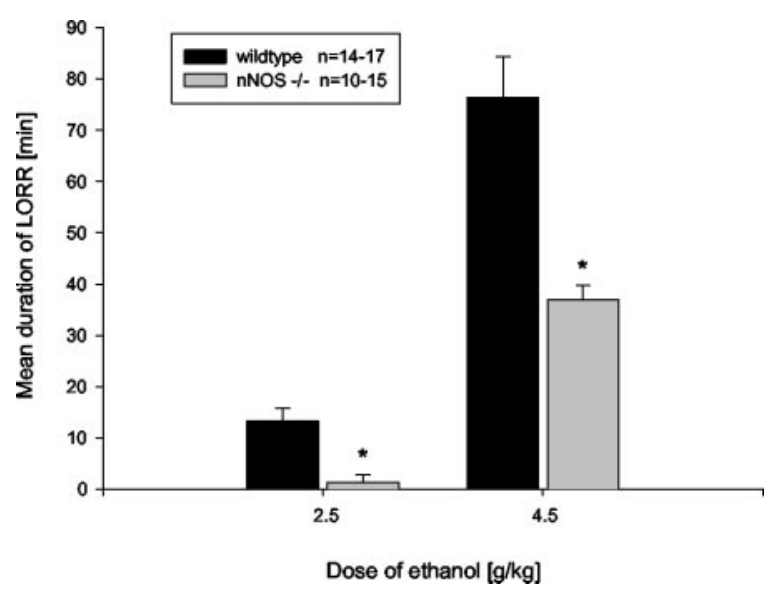

Figure 5. Sensitivity to the hypnotic effect of ethanol assessed by the measurement of loss of righting response $(L O R R)$ in wild-type and nNOS $-/-$ mice. Ethanol (2.5 and $4.5 \mathrm{gm} / \mathrm{kg}$, i.p.) was injected, and the time to regain the righting effect was measured. Each value is the mean + SE. ${ }^{*} p<0.05$ indicates a significant difference from wild-type mice.

than wild-type mice $\left(2.5 \mathrm{gm} / \mathrm{kg}, t_{(23.6)}=3.76, p=0.0010 ; 4.5\right.$ $\left.\mathrm{gm} / \mathrm{kg}, t_{(16.4)}=4.44, p=0.0004\right)$ (Fig. 5).

For studying tolerance, we used a sensitive quantifiable method that could detect rapid changes in ethanol effects. We examined the hypothermic effect of peripherally administered ethanol and the rapid development of tolerance to this effect (Crabbe et al., 1979). Acute ethanol injection (3.5 gm/ kg, i.p.) produced a significant decrease in body temperature in wild-type $\left(t_{(8)}=5.00\right.$; $p=0.0010)$ and nNOS $-/-\left(t_{(8)}=3.45 ; p=0.0087\right)$ animals 30 min after injection (Fig. $6 A, B)$. A comparable decrease in body temperature was not observed in wild-type mice $24 \mathrm{hr}$ later when a second ethanol injection $(3.5 \mathrm{gm} / \mathrm{kg}$, i.p. $)$ was given $\left(t_{(8)}=1.12\right.$; $p=.2956)$, indicating the development of rapid tolerance. However, in nNOS $-/-$ mice, a strong decrease in body temperature after the second ethanol injection still occurred $\left(t_{(8)}=3.51 ; p=\right.$ 0.0080 ), indicating resistance to the development of tolerance in the knock-out animals (Fig. 6A,B).

To rule out the possibility that differences in alcohol consumption, sensitivity, and tolerance between nNOS -/- and wild-type mice underlies increased ethanol metabolism, we tested blood alcohol elimination after the injection of a high dose of ethanol (3.5 gm/kg, i.p.) (Fig. 7). Blood alcohol levels reached a maximum after $30 \mathrm{~min}$ after injection $(\sim 300 \mathrm{mg} / \mathrm{dl})$. Speed of elimination of this maximum concentration was measured in each animal by fitting a linear regression line and determining its slope. Comparing both groups of animals with respect to elimination speed defined in this way gave no significant difference in mean $\left(t_{(7.53)}=1.12 ; p=0.344\right)$. Furthermore, the elimination rates did not differ between the genotypes (nNOS $-/-, 0.58 \pm 0.05 \mathrm{mg}$ of ethanol per milliliter of blood per hour; wild-type, $0.55 \pm 0.04 \mathrm{mg}$ of ethanol per milliliter of blood per hour).

\section{DISCUSSION}

\section{Alcohol drinking in nNOS -/- mice}

We studied voluntary alcohol consumption in nNOS knock-out mice. Alcohol intake and preference in nNOS $-/-$ mice did not differ from that in wild-type animals at low ethanol concentrations (2-4\%); however, a pronounced difference was found at higher ethanol concentrations $(8-16 \%)$. Thus, nNOS $-/-$ mice consumed approximately sixfold more alcohol than wild-type 

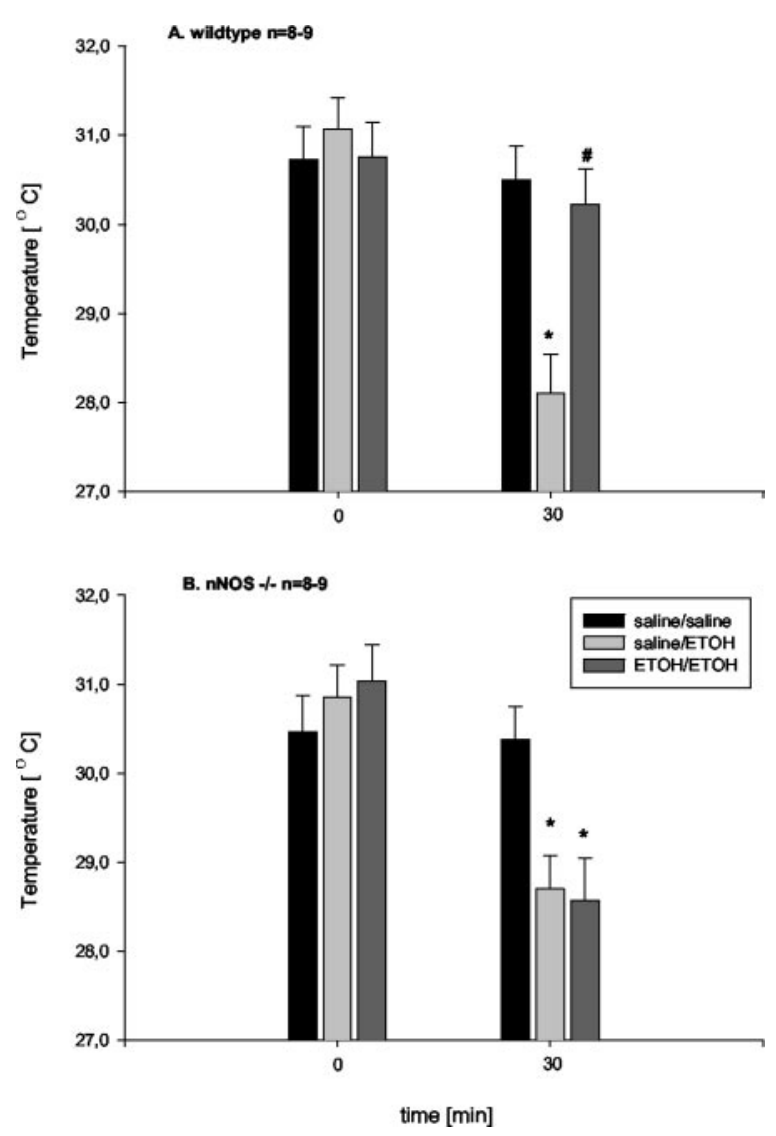

Figure 6. Development of rapid tolerance to ethanol-induced hypothermia in wild-type mice $(A)$ but not in nNOS knock-out mice $(B)$. The figure represents the effects of a second ethanol injection $(3.5 \mathrm{gm} / \mathrm{kg}$, i.p.) in animals that received $24 \mathrm{hr}$ before the first ethanol injection (3.5 $\mathrm{gm} / \mathrm{kg}$, i.p.). Bars represent the mean $+\mathrm{SE}$ of the ventral surface temperature measured by an infrared thermometer in eight to nine mice per group. ${ }^{*} p<0.05$ indicates significant differences from the basal values (time point 0 ).

mice, and some knock-out mice reached pharmacologically meaningful blood alcohol levels (up to $58 \mathrm{mg} / \mathrm{dl}$ ). Although these data show that loss of a functional nNOS gene results in increased alcohol intake, it should be mentioned that the wild-type animals, which had a predominant C57BL/6J background, had a very low alcohol intake, which resulted in no meaningful blood alcohol levels. It is very likely that a gene-environment effect as described by Crabbe et al. (1999) had contributed to this unusual low alcohol intake in the wild-type animals.

In rodents, alcohol intake is partially dependent on its flavor. Thus, alcohol taste has a sweet-bitter component, and the proclivity to drink alcohol is associated with elevated sweet preferences and/or lower aversion to a bitter taste. This has been shown previously in C57BL/6J mice compared with $129 / \mathrm{J}$ mice: the higher alcohol intake by $\mathrm{C} 57 \mathrm{BL} / 6 \mathrm{~J}$ mice depends, in part, on higher hedonic attractiveness of its sweet taste component (Bachmanov et al., 1996). C57BL/6J mice have an additional tendency to avoid bitter quinine less than do 129 mice (Bachmanov et al., 1996). Different concentrated sucrose solutions were offered in a free-choice paradigm before and after alcohol self-administration to our animals. nNOS $-/-$ mice showed a higher sucrose preference than wild-type mice, especially at an intermediate sucrose concentration. Other taste differences could not be observed; there was a clear taste aversion for bitter-tasting quinine solutions

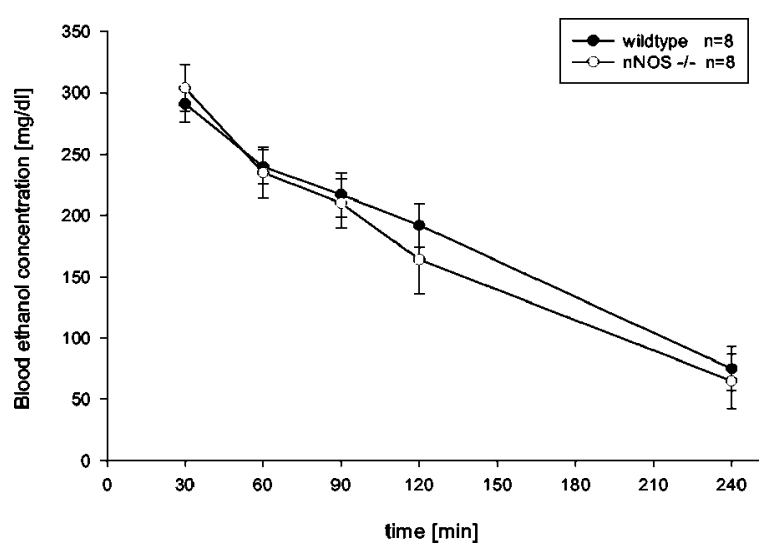

Figure 7. Blood ethanol elimination curve in nNOS knock-out and wild-type mice. nNOS $-/-$ and wild-type mice received an intraperitoneal injection of $3.5 \mathrm{gm} / \mathrm{kg}$ ethanol, and blood samples were taken from the tail vein at different time points. Values are means $\pm \mathrm{SE}$ in milligrams of ethanol per deciliters of blood.

in nNOS $-/-$ mice, as well as wild-type mice. In other species, an association of preference for sweet-tasting solutions and preference for alcohol was also demonstrated. Alcohol-preferring rats show higher sweet solution intake than alcohol nonpreferring rats (Stewart et al., 1994), and, in a clinical study, Kampov-Polevoy et al. (1997) demonstrated that a majority $(>80 \%)$ of their alcoholic patients were sweet likers, i.e., preferred high sucrose concentrations. However, it is unlikely that $\mathrm{nNOS}-/-$ mice consumed more alcohol than wild types because they are sweet likers, because initial alcohol preference at lower concentrated ethanol solutions did not differ between nNOS $-/-$ mice and wild-type mice despite the fact that ethanol solutions up to a concentration of $6 \%$ are usually preferred by rodents, probably because of their sweet-like taste component (Li et al., 2001).

The present findings in nNOS $-/-$ mice on alcohol intake and preference are in stark contrast to drinking data obtained in pharmacological studies using nonselective NOS inhibitors. Administration of either L-NNA or L-NAME compounds, which inhibit all isoforms of NOS, attenuated alcohol consumption in two lines of alcohol-preferring rats (Rezvani et al., 1995), in Sprague Dawley rats selected for high alcohol intake (Calapai et al., 1996), and in rats that drank chronically alcohol in combination with L-NNA (Lallemand and De Witte, 1997). On the other hand, a knock-out of the nNOS gene leads to a marked enhancement of alcohol consumption. How can this discrepancy be explained? Interestingly, we found that repeated L-NAME injections significantly reduced alcohol intake and preference in wild-type mice, as well as nNOS $-/-$ mice. From these experiments, we have to conclude that the effects of nonselective NOS inhibitors on alcohol intake are not solely mediated by nNOS. Several splice variants of the nNOS gene exist, and, in the nNOS $-/-$ mice, the $\alpha$ variant, which accounts for the great majority of catalytic activity in the brain (Huang et al., 1993), is completely absent. Our in situ hybridization data, however, show that $\mathrm{nNOS} \beta$ and $\gamma$ splice variants are still present in knock-out animals, and $n N O S \beta$ is even slightly increased, in particular within striatal and cortical tissue, which is in accordance with a previous study (Eliasson et al., 1997). Although it is assumed that the $\beta$ and $\gamma$ splice variants of the nNOS gene are also blocked by nonselective NOS inhibitors, it is not clear whether inhibition of this small rest activity of the nNOS $\beta$ and $\gamma$ variants can account for the reduction in alcohol consumption after 
treatment with NOS inhibitors in the knock-out mice. Alternatively, inhibition of the eNOS and/or iNOS isoforms, which are also targets of nonselective NOS inhibitors, could contribute to the alcohol suppressant effect of these compounds. Furthermore, it might well be that the effect of NOS inhibitors on alcohol consumption is mainly mediated via the periphery because NO is a potent vasodilator and NOS inhibitors can drastically influence blood pressure (Ribeiro et al., 1992), which in turn can influence the pharmacokinetic properties for ethanol (Vassiljev et al., 1998). Thus, it is possible that the reduction seen in alcohol intake after NOS blockade is attributable to these unspecific effects of NOS inhibitors. This suggestion is further supported by our observation that, at the onset of the L-NAME treatment, nonspecific effects such as sedation, reduction in total fluid intake, and food consumption were observed. Our observations are in line with previous reports showing that intake of water is modulated by NO (Calapai et al., 1992) and that food consumption can be reduced by blocking NO formation with antagonists of NOS (Morley and Flood, 1991; Squadrito et al., 1993).

\section{Alcohol sensitivity and tolerance in nNOS - /- mice}

In another line of experiments, we investigated whether nNOS -/mice and wild-type mice would differ in terms of sedative-hypnotic effects of an acutely administered high dose of ethanol. This study was of interest because clinical research has proposed that initial sensitivity to ethanol may be negatively correlated with subsequent ethanol intake (Schuckit, 1994; Schuckit and Smith, 1996). In mice, such a correlation has also been found, e.g., in protein kinase A mutant mice high alcohol intake was associated with low sensitivity to ethanol-induced sedation (Thiele et al., 2000). Our studies on loss of righting reflex showed that nNOS $-/-$ mice regained their righting reflex much faster than wild-type mice. Thus, nNOS -/mice are less sensitive to intoxicating blood alcohol levels and drink subsequently more alcohol than wild-type mice. Again, these findings are in contrast to a previous study showing that pretreatment with L-NAME delayed the onset of ethanol-induced loss of righting reflex and increased the duration of the loss of righting reflex (Adams et al., 1994). However, in this study, a very high dose range of L-NAME (30-100 mg/kg, s.c.) was used, which might have produced unspecific effects as observed in our drinking studies.

In addition, we studied the development of rapid tolerance to the hypothermic effect of ethanol. It has been demonstrated that tolerance to the hypothermic effect of a single ethanol injection during administration of an equivalent dose $24 \mathrm{hr}$ later occurs in mice (Crabbe et al., 1979). Interestingly, nNOS $-/-$ mice did not develop rapid tolerance to ethanol-induced hypothermia, which is in line with a previous report showing that the NOS inhibitor L-nitroarginine blocked the development of rapid tolerance to the motor incoordinating effect of ethanol (Khanna et al., 1995). It is possible that the nNOS $-/-$ mice showed high ethanol intake, less sensitivity to the sedative-hypnotic effects of ethanol, and no rapid tolerance to ethanol because of an increased rate of alcohol metabolism. However, this seems unlikely, because the nNOS -/mice and wild-type mice did not differ in plasma ethanol concentrations during the whole time course of $4 \mathrm{hr}$ after acute ethanol injection.

In summary, we showed that nNOS is involved in the regulation of several neurobehavioral effects of alcohol, including alcohol sensitivity, tolerance, and reinforcement. In particular, alterations in the nNOS gene can lead to changes in alcohol drinking behavior. Thus, nNOS knock-out mice have a much higher voluntary alcohol intake than wild-type mice. On the other hand, these knock-out animals are less sensitive to the sedative hypnotic effects of a high ethanol dose, which might promote high alcohol intake. However, it should be emphasized that the conclusions of behavioral alcohol studies with mouse mutants are limited to the specific genetic background used and that environmental factors can also influence the behavioral phenotype. With this limitations in mind, we suggest that alterations in the nNOS gene may constitute a genetic risk factor for the vulnerability of high voluntary alcohol intake and subsequent alcohol dependence. Our study further suggests that the selective pharmacological targeting of the nNOS gene or its product may not lead to a promising intervention strategy of alcohol abuse.

\section{REFERENCES}

Adams ML, Meyer ER, Sewing BN, Cicero TJ (1994) Effects of nitric oxide-related agents on alcohol narcosis. Alcohol Clin Exp Res 18:969-975.

Azad SC, Marsicano G, Eberlein I, Putzke J, Zieglgänsberger W, Spanagel R, Lutz B (2001) Differential role of the nitric oxide pathway on $\Delta$-THC-induced central nervous system effects in the mouse. Eur J Neurosci 13:561-568.

Bachmanov AA, Tordoff MG, Beauchamp GK (1996) Ethanol consumption and taste preferences in C57BL/6J and 129/J mice. Alcohol Clin Exp Res 20:201-206.

Bredt DS, Snyder SH (1994) Nitric oxide: a physiologic messenger molecule. Annu Rev Biochem 6:175-195.

Bredt DS, Hwang PM, Snyder SH (1990) Localization of nitric oxide synthase indicating a neural role for nitric oxide. Nature 347:768-770.

Calapai G, Squadrito F, Altavilla D, Zingarelli B, Campo GM, Cilia M, Caputi AP (1992) Evidence that nitric oxide modulates drinking behaviour. Neuropharmacology 31:761-764.

Calapai G, Mazzaglia G, Sautebin L, Costantino G, Marciano MC, Cuzzocrea S, Di R, Caputi AP (1996) Inhibition of nitric oxide formation reduces voluntary ethanol consumption in the rat. Psychopharmacology 125:398-401.

Chandler LJ, Guzman NJ, Sumners C, Crews FT (1994) Magnesium and zinc potentiate ethanol inhibition of $N$-methyl-D-aspartate-stimulated nitric oxide synthase in cortical neurons. J Pharmacol Exp Ther 271:67-75.

Chandler LJ, Sutton G, Norwood D, Sumners C, Crews FT (1997) Chronic ethanol increases $N$-methyl-D-aspartate-stimulated nitric oxide formation but not receptor density in cultured cortical neurons. Mol Pharmacol 51:733-740.

Crabbe JC, Rigter H, Uijlen J, Strijbos C (1979) Rapid development of tolerance to the hypothermic effect of ethanol in mice. J Pharmacol Exp Ther 208:128-133.

Crabbe JC, Wahlsten D, Dudek BC (1999) Genetics of mouse behavior: interactions with laboratory environment. Science 284:1670-1672.

Eliasson MJ, Blackshaw S, Schell MJ, Snyder SH (1997) Neuronal nitric oxide synthase alternatively spliced forms: prominent functional localizations in the brain. Proc Natl Acad Sci USA 94:3396-3401.

Harris RA, McQuilkin SJ, Paylor R, Abeliovich A, Tonegawa S, Wehner JM (1995) Mutant mice lacking the $\gamma$ isoforms of protein kinase C show decreased behavioral actions of ethanol and altered function of $\gamma$-aminobutyrate type A receptors. Proc Natl Acad Sci USA 92:3658-3662.

Holm S (1979) A simple sequentially rejective multiple test procedure. Scand J Statist 6:65-70.

Huang PL, Lo EH (1998) Genetic analysis of NOS isoforms using nNOS and eNOS knockout animals. Prog Brain Res 118:13-25.

Huang PL, Dawson TM, Bredt DS, Snyder SH, Fishman MC (1993) Targeted disruption of the neuronal nitric oxide synthase gene. Cell 75:1273-1286.

Itzhak Y, Martin JL (2000) Blockade of alcohol-induced locomotor sensitization and conditioned place preference in DBA mice by 7-nitroindazole. Brain Res 858:402-407.

Itzhak Y, Ali SF, Martin JL, Black MD, Huang PL (1998) Resistance of neuronal nitric oxide synthase-deficient mice to cocaine-induced locomotor sensitization. Psychopharmacology 140:378-386.

Kampov-Polevoy AB, Garbutt JC, Janowsky D (1997) Evidence of preference for a high concentration sucrose solution in alcoholic men. Am J Psychiatry 154:269-270.

Khanna JM, Morato GS, Shah G, Chau A, Kalant H (1993) Inhibition of nitric oxide synthase impairs rapid tolerance to ethanol. Brain Res Bull 32:43-47.

Khanna JM, Morato GS, Chau A, Shah G (1995) Influence of nitric oxide synthase inhibition on the development of rapid tolerance to ethanol. Brain Res Bull 37:599-604.

Kolesnikov YA, Pick CG, Ciszewska G, Pasternak GW (1993) Blockade 
of tolerance to morphine but not to kappa opioids by a nitric oxide synthase inhibitor. Proc Natl Acad Sci USA 90:5162-5166.

Kolesnikov YA, Pan YX, Babey AM, Jain S, Wilson R, Pasternak GW (1997) Functionally differentiating two neuronal nitric oxide synthase isoforms through antisense mapping: evidence for opposing NO actions on morphine analgesia and tolerance. Proc Natl Acad Sci USA 94:8220-8225.

Lallemand F, De Witte P (1997) L-NNA decreases cortical vascularization, alcohol preference and withdrawal in alcoholic rats. Pharmacol Biochem Behav 58:753-761.

Li T-K, Spanagel R, Colombo G, McBride WJ, Porrino LJ, Suzuki T, Rodd-Henricks ZA (2001) Alcohol reinforcement and voluntary ethanol consumption. Alcohol Clin Exp Res 25:117-126.

Morley JE, Flood JF (1991) Evidence that nitric oxide modulates food intake in mice. Life Sci 49:707-711.

Putzke J, Seidel B, Huang PL, Wolf G (2000) Differential expression of alternatively spliced isoforms of neuronal nitric oxide synthase (nNOS) and $N$-methyl-D-aspartate receptors (NMDAR) in knockout mice deficient in $\mathrm{nNOS} \alpha\left(\mathrm{nNOS} \alpha \Delta^{/ \Delta}\right.$ mice). Mol Brain Res 85:13-23.

Rezvani AH, Grady DR, Peek AE, Pucilowski O (1995) Inhibition of nitric oxide synthesis attenuates alcohol consumption in two strains of alcohol-preferring rats. Pharmacol Biochem Behav 50:265-270.

Ribeiro MO, Antunes E, de Nucci G, Lovisolo SM, Zatz R (1992) Chronic inhibition of nitric oxide synthesis. A new model of arterial hypertension. Hypertension 20:298-303.
Schuckit MA (1994) Low level of response to alcohol as a predictor of future alcoholism. Am J Psychiatry 151:184-189.

Schuckit MA, Smith TL (1996) An 8-year follow-up of 450 sons of alcoholic and control subjects. Arch Gen Psychiatry 53:202-210.

Spanagel R, Putzke J, Zieglgänsberger W, Stefferl A, Schöbitz B (1996) Acamprosate and alcohol. II. Effects on alcohol withdrawal in the rat. Eur J Pharmacol 305:45-50.

Squadrito F, Calapai G, Cucinotta D, Altavilla D, Zingarelli B, Ioculano M, Urna G, Sardella A, Campo GM, Caputi AP (1993) Anorectic activity of $N^{\mathrm{G}}$-nitro-L-arginine, an inhibitor of brain nitric oxide synthase, in obese Zucker rats. Eur J Pharmacol 230:125-128.

Stewart RB, Russell RN, Lumeng L, Li T-K, Murphy JM (1994) Consumptions of sweet, salty, sour, and bitter solutions by selectively bred alcohol preferring and alcohol-nonpreferring lines of rats. Alcohol Clin Exp Res 18:375-381.

Tabakoff B, Hoffman PL (1996) Alcohol addiction: an enigma among us. Neuron 16:909-912.

Thiele TE, Willis B, Stadler J, Reynolds JG, Bernstein IL, McKnight GS (2000) High ethanol consumption and low sensitivity to ethanolinduced sedation in protein kinase $A$ mutant mice. $J$ Neurosci 20:RC75(1-6).

Tsai G, Coyle JT (1998) The role of glutamatergic neurotransmission in the pathophysiology of alcoholism. Annu Rev Med 49:173-184.

Vassiljev V, Vali M, Pokk P (1998) Effects of nitric oxide synthase inhibitors L-NAME and L-NOARG on behavioural signs of ethanol withdrawal. Med Sci Res 27:409-410. 